\title{
Onomastique, vacances et autoreprésentation dans Noëlle à Cuba de Pierre Karch
}

\author{
Mary Ellen Ross
}

(Université de Victoria)

Quoique Noëlle à Cuba raconte les vacances de Noël d'une trentaine de touristes, québécois pour la plupart, sous le soleil de Cuba, cet ouvrage de Pierre Karch n'a rien de banal: plutôt qu'un roman d'évasion, il s'agit d'un "roman sur l'évasion." Comme le note Pierre Hébert, l'intérêt et la complexité du roman dépendent justement du fait que Noëlle à Cuba n'est pas uniquement le récit des aventures d'un groupe de vacanciers qui fuient les neiges septentrionales. En effet, Hébert insiste sur une autre dimension du roman, la rencontre dans Noëlle à Cuba de l'art et du voyage, de la fiction et des vacances. ${ }^{2}$

Noëlle à Cuba pose systématiquement la correspondance de la récréation propre aux vacances et de la re-création de soi qui permet au touriste de s'inventer un nouvel être. Ainsi, le personnage Liljana constate que le voyage permet de refaire l'identité, les vacances n'étant rien de moins qu'une fiction forgée par celui ou celle qui est loin de son foyer: "S'il est vrai qu'en vacances on laisse tomber son masque, c'est souvent pour en laisser paraître un autre." ${ }^{3}$ Mais c'est à un autre personnage, l'écrivain anonyme également en vacances, de développer ce parallélisme dans son journal:

Les vacances appartiennent, comme le roman, au domaine du rêve que le jour interrompt à son pire ou à son meilleur moment.

Les vacances? Cela n'existe pas vraiment; c'est une fiction qu'on se crée, qu'on s'invente à mesure, qu'on modifie en cours de route et qu'on a du mal à reconnaître pour sienne si plus tard on tente de la reconstruire à partir des quelques objets preuves qui nous sont restés.

Les vacances, j'entends surtout celles passées à l'étranger, permettent à chacun d'être 
ce qu'il voudrait et de vivre ses phantasmes à l'intèrieur de parenthèses bien définies, rassurantes donc, car on sait qu'il est impossible de les déborder. Loin du décor familier, on cesse de se conformer à l'image que les autres se font de soi, on lâche son fou, tout en sachant que cela est sans conséquence, parce que temporaire, la routine et les habitudes nous attendant au retour. Et, comme personne n'est là pour témoigner contre soi, on peut s'imaginer et faire croire aux autres tout ce que l'on voudra, les seules limites étant celles de son imagination. Belles, cent fois belles les vacances où l'on se re-crée ainsi! Cela ne dérange ni Dieu ni personne. Au contraire, tout le monde s'y attend parce que chacun joue, sur ce théâtre, le jeu de la déception (p. 360).

Ce passage, qui confond volontiers vacances et roman des vacances, orientera notre lecture de Noëlle à Cuba. S'il va sans dire que les vacances décrites dans Noëlle à Cuba sont fictives, il faut reconnaître de plus que ce roman met l'accent sur le caractère irréel des épisodes racontés, problématisant "l'identité" des personnages. Comme nous le verrons, l'onomastique constitue l'une des techniques de base employées dans Noëlle à Cuba pour poser l'équivalence de vacances et d'art romanesque en tant que création d'un personnage, d'un rôle ou d'un "masque:" la majorité des noms de personnages dans Noëlle à Cuba, loin de suggérer une identité stable et "vraisemblable" propre au personnage, présentent un intertexte mythologique, effectuent un jeu de langage ou permettent même dans certains cas une métamorphose fantastique, procédés éminemment autoreprésentatifs et ludiques. ${ }^{4}$ Ainsi, l'onomastique va de pair avec les nombreux passages du roman qui mettent en lumière le "rôle" du personnage sur le "théâtre" des vacances: la nature ludique et autoréférentielle de l'onomastique dans Noëlle à Cuba insiste sur le personnage en tant qu'être, non en chair et en os mais plutôt fait de plume et d'encre, être de vacances et de fiction. ${ }^{5}$ 
Sur les vingt-neuf touristes en vacances à l'Hôtel Marazul dans Noëlle à Cuba, un peu plus de la moitié, c'est-à-dire seize, n'ont qu'un prénom. Les noms de famille qui figurent dans Noëlle à Cuba (Branchu, Camelot, Duressec, Lannes, McDonald, Mollard, RinseauDesprez, Rolland, Vervier) sont presque tous des noms parlants, d'habitude humoristiques, comme le sont aussi deux prénoms nonmythologiques (Astrid, Noëlle). D'autre part, cinq prénoms (Adèle "Lèda," Daphné, Eurydice, Icare, Paul "Apollon") se rattachent, directement ou par le biais de l'anagramme ou de l'homonymie partielle, à la mythologie grecque; les touristes qui portent ces noms vivent des épisodes ressemblant de près aux aventures de leurs contreparties antiques. ${ }^{6}$ Les personnages cubains de Noëlle à Cuba, moins nombreux que les touristes étrangers, présentent néanmoins une distribution onomastique similaire: le prénom "Mariposa" et le sobriquet "La Veuve Noire" relèvent du jeu de langage, tandis que trois Cubains (Chango, Dédalo, Ochun) ont un prénom mythologique. Aucun personnage cubain n'a de nom de famille. ${ }^{7}$ Nous examinerons d'emblée les prénoms et les noms de famille ludiques qui, en insistant sur un trait capital du personnage, constituent un jeu de langage, pour passer ensuite aux noms qui évoquent un intertexte mythologique.

\section{Prénoms et noms de famille ludiques}

Selon Philippe Hamon, le récit "classique" (ou lisible), qui a horreur du vide sémantique et, par conséquent, du nom privé de sens, ne précise le nom du personnage normalement qu'après une description, un portrait. ${ }^{8}$ On se rend compte en analysant l'onomastique dans Noëlle à Cuba que dans ce texte le contraire se produit d'habitude: non seulement ce roman fournit-il peu de portraits, mais aussi c'est le nom qui, le plus souvent, qualifie l'individu, soulignant une seule caractéristique qui le définit ou bien annonçant le rôle qu'il jouera dans le récit. Force est de reconnaitre une première fonction, des plus pratiques, de l'onomastique dans Noëlle à Cuba: le fait d'assigner des noms parlants aux personnages permet au lecteur de distinguer cette panoplie d'êtres les uns des autres. Le cas d'Astrid illustre ce point. Les dons de cette femme, qui s'intéresse à l'horoscope des autres touristes et qui réussit en fait à prédire la mort 
d'un vacancier, sont annoncés par son nom; comme elle le reconnait elle-même, Astrid est celle qui lit l'avenir dans les astres:

Non, ma mère n'avait jamais cru si bien faire en me nommant Astrid. Ce sont les astres que je consulte (p. 188).

De plus, le texte dévoile ici, comme il arrive souvent pour ce qui est de l'onomastique dans Noëlle à Cuba, son propre fonctionnement, dans la mesure où le nom qui joue sur le langage est commenté explicitement par un personnage ou par le narrateur.

Il en va de même pour Noëlle, baptisẻe ainsi pour permettre le jeu de mots du titre, titre qui est, comme le note Pierre Léon, un "ressort poétique," plutôt qu'un indice de l'importance de Noëlle comme personnage. ${ }^{9}$ Noëlle elle-même trouve ce jeu de langage assez évident, puisqu'elle pense, au moment où elle dit son nom à un autre touriste, "S'il me demande si je suis en vacances de Noël, c'est bien simple, je le mords!" (p. 79)

Dans le cas des noms de famille ludiques, le jeu de langage est souvent manifeste, quoique ni les personnages ni le narrateur ne le commentent de façon explicite. Ainsi, Carmen et Michael Duressec portent ce nom parce qu'ils sont de la deuxième "vague" de touristes à l'Hôtel Marazul:

C'était, le matin, une marée de valises et de sacs lourds de coquillages et de bouteilles de rhum ou de liqueurs Havana Club qui descendaient vers la sortie avant d'être lancès, sans cérémonie, dans le compartiment à bagages du bus qui se rendrait à l'aéroport, quatre heures avant le départ de l'avion. Et c'était, en fin d'après-midi, une nouvelle marée de sacs et de valises qui montaient vers les chambres rafraîchies où on les déposait avec soin au pied des lits.

Cette fin de semaine-là, (...) Michael et Carmen Duressec avaient été bien étonnés d'être les 
seuls à descendre au Marazul, se demandant ce qui leur avait valu cette distinction (p. 225).

Les jeux onomastiques se multiplient: le nom de famille de Lise Camelot commente, dans la mesure où le substantif "camelot" signifie un vendeur, souvent de journaux, la seule caractéristique importante du personnage, l'habitude cocasse qu'elle a de lire pendant ses vacances les journaux des six derniers mois. Fait significatif, on voit Enid Rinseau-Desprez, auteur de contes tout à fait anodins (rinse+eau; sot) pour la première fois lorsqu'elle est devant la porte des toilettes à l'aéroport (p. 20). Pour sa part, Diane-D. Mollard, dont le nom insolite suggère la paresse (molle+lard) et le crachat (mollard), néglige son enfant Sacha et déteste les hommes, tout en s'étendant "mollement" sur le sable (p. 201). Et si le prénom d'Eurydice Branchu laisse prévoir un intertexte mythologique dont il sera question plus tard, son nom de famille révèle que cette grosse mère et grand-mère est un arbre aux nombreuses «branches:»

- Les enfants ne s'ennuient jamais de leurs parents. Pas pour longtemps en tout cas.

(Eurydice) pensait aux fruits de l'arbre qui tombent tout autour et roulent plus loin et à l'arbre trop gros pour bouger et qui les voit partir, impuissant à les retenir (p. 163).

D'ailleurs, le nom de famille d'Eurydice suggère une deuxième lecture, dans la mesure où ce personnage immobile est "branchée," toujours au courant de ce qui se passe à l'hôtel. Quand Noëlle cherche Icare, c'est à Eurydice qu'elle demande des renseignements:

(Noëlle) courut jusqu'à la salle à manger. Il n'y était pas. Mais Eurydice y était. S'il était venu. elle le saurait. Elle savait tout. Elle ne bougeait presque pas de la journée et tout le monde passait devant elle. ${ }^{10}$

Chez les personnages cubains, deux cas qui se ressemblent à certains égards sont ceux de la Veuve Noire et de Mariposa. On 
pourrait parler ici d'une onomastique de la Cubaine-insecte, dans la mesure où la Veuve Noire ressemble étrangement à l'araignée qui porte ce nom, tandis que Mariposa, dont le nom signifie "papillon" en espagnol, semble effectivement se métamorphoser à plusieurs reprises en papillon. Laraignée qu'on appelle la Veuve Noire, qui fréquente des endroits sombres, doit son nom à la croyance erronée qu'elle dévore souvent le mâle après l'accouplement. ${ }^{11}$ Or, Icare faillit se faire tuer par un agent de la police militaire cubaine lorsque, fasciné, il accoste la Veuve Noire: "On lui aurait dit que son baiser était aussi mortel que sa morsure qu'il (Icare) n'aurait pas moins voulu s'approcher d'elle" (p. 51). D'ailleurs, la deuxième rencontre d'Icare et de la Veuve Noire a lieu dans un caveau de cimetière (pp. 90-93). Ainsi, le nom de la Veuve Noire, suggérant la femme-araignée au venin fatal, évoque un érotisme menaçant, qui n'est pas sans rappeler celui du récit fantastique. ${ }^{12}$

Si la Veuve Noire ressemble à une araignée, Mariposa est un personnage plus inquiétant considéré selon le point de vue du récit "réaliste," puisqu'elle semble devenir papillon par moments. Le narrateur indique quaprès une conversation avec Icare, Mariposa "s'envola, comme elle était venue," verbe lourd de signification ( $p$. 106). D'ailleurs, ce personnage au nom significatif semble même penser comme le ferait un papillon:
Mariposa se dit que c'était Marco qui l'avait renseigné (Icare). Un vaurien, Marco. Un filou et un mouchard sans intèrêt ni envergure. Icare, c'était une autre paire d'ailes (p. 105).

La transformation ludique du cliché "C'est une autre paire de manches" se fait à partir du nom du personnage; c'est en fait son prénom qui détermine l'identité et le point de vue personnel de Mariposa. ${ }^{13}$ De même, Mariposa visite avec Icare la salle de papillons du Musée des sciences naturelles, où Icare se demandera s'il a lui-même des ailes. ${ }^{14}$ Ailleurs, Mariposa elle-même prètend avoir des ailes (p. 129), tandis qu'Icare la traite de "chenille" et croit même la voir se métamorphoser en papillon de nuit et se mettre à tourner autour d'un lampadaire en attendant qu'il fasse jour et que l'appellent les fleurs comme autant de soleils" (p. 144). Puisque 
Mariposa réalise ainsi le sens de son prénom, on ne peut manquer de penser à elle à la fin du roman, lorsqu'un papillon s'écrase contre la porte vitrée de l'aéroport que vient de passer Icare (p. 391-392). Ainsi, l'onomastique permet un fantastique discret, qu'il s'agisse de la femme-araignée à la sensualité menaçante ou de la jeune fillepapillon qui suit Icare et qui voudrait qu'il l'aime. ${ }^{15}$

\section{Personnages à prénom mythologique ${ }^{16}$}

Deux personnages cubains, Chango et Ochun, portent des noms de divinitès afro-cubaines. ${ }^{17}$ Ces deux danseurs participent au spectacle de la "Nuit afro-cubaine" et en fait, l'onomastique ne distingue pas le rôle joué par Chango et Ochun lors du spectacle touristique, celui du dieu et de la déesse dont ils portent le nom, et leur "vraie" identité de tous les jours. Effectivement, Chango et Ochun n'ont pas de nom ni d'être distincts de ceux des divinités qu'ils incarnent lors du spectacle: le narrateur se réfère à Ochun comme "la déesse de l'amour," tandis que Chango incarne "l'homme dans ce qu'il a de plus brutal, de plus bestial, de plus instinctif" (p. 194). Ce chevauchement du mythe et de la "réalité" fictive, du rôle joué dans un spectacle et de l'identité chez Ochun et Chango, s'insère dans une série d'allusions autoreprésentatives au théâtre et au rôle des personnages de Noëlle à Cuba. ${ }^{18}$

Les cinq noms mythologiques qui restent se rattachent tous aux légendes de l'Antiquité grecque. Il s'agit de ces noms motivés qui, selon Hamon, "fonctionnent (...) comme des condensés de programmes narratifs, anticipant et laissant préfigurer le destin même des personnages (nomen-numen) qui les portent" (Hamon, p. 150). Ainsi, Daphné, dont le nom signifie en grec "laurier," revit en quelque sorte l'épisode raconté par Ovide, où la prière de la nymphe, qui fuit le désir d'Apollon est exaucée: elle est changée en laurier. ${ }^{19}$ Le texte commente ici encore l'intertexte que laisse prévoir l'onomastique: le nouveau mari de Daphné, Paul (Apollon) jure qu'il aura sa femme, "devait-elle se métamorphoser en laurier" (p. 57). D’ailleurs, le narrateur souligne le fait que Paul joue un rôle, suivant un script, en séduisant sa femme: 
Et Paul n'était pas fatigué. Une force divine le pénétrait, capable d'assouvir les nymphes mêmes. Mais, autant le décor de la veille l'avait intimidé par ce qu'il avait de somptueux, autant celui-ci le repoussait par sa rigueur janséniste. Il lui fallait un théâtre où il puisse donner sa pleine mesure: la grotte de Thétys peut-être ou le mont Olympe (p. 58).

Comme chez la Veuve Noire et Mariposa, nous avons affaire à un fantastique de l'onomastique, non seulement à cause de la thématique "magique" de la métamorphose chez Ovide, mais aussi puisque le sort du personnage Daphné chez Karch est étroitement lié à celui des fleurs qui l'entourent et surtout à celui d'un laurierrose. Tout de suite après la cérémonie de noces, avant même le voyage à Cuba, Daphné paraît aussi "défraîchie" que son corsage ( $\mathrm{p}$. 29). Et, voyant Daphné triste après sa nuit de noces, Icare lui offre un laurier-rose. Il est significatif quau moment où cette action d'Icare rappelle le mythe grec, d'autant plus qu'il parle à Daphné d'une voix "comme la harpe d'Apollon" (p. 71), Daphné est consciente du fait qu'elle joue un rôle:

Daphné sentait qu'elle jouait un personnage, qu'elle interprétait un rôle, qu'on lui soufflait chaque réplique à mesure qu'avançait l'action et cela lui plut tellement qu'elle se prêta volontiers au jeu:

-Seriez-vous, par hasard, le Chevalier au laurier-rose?

-Pour vous servir, ma Dame (p. 71).

L'importance du laurier-rose, évoqué ici par l'offrande d'Icare, s'accroît au moment où Ana décide de peindre Daphné, qui tient cette fleur. Dès lors, c'est le laurier-rose qui se métamorphose, adoptant par une sorte de contamination métonymique ${ }^{20}$ les traits de Daphné: 
(Ana) s'aperçut que la fleur qu'elle avait commencé de peindre empruntait maintenant, à la joue et à l'oreille de Daphné, un teint nouveau plus vif, plus sensuel comme celui des bergères de Fragonard (p. 188).

La ressemblance de Daphné et de la fleur se fait de plus en plus inquiètante, de sorte qu'on ne saurait attribuer toutes les transformations de la fleur aux effets changeants de la lumière ni au langage figuré:

(...) la fleur était tellement belle, souriante, douce et parfumée que Daphné la porta de nouveau à ses lèvres.

De blanche qu'elle ètait un instant plus tôt, elle devint carmin comme si le sang lui montait aux pétales. (...) La fleur parlait à Daphné comme une soeur, en lui souriant, sans qu'il passe entre ses pétales ouverts plus qu'un léger parfum qui l'intoxiquait. Et Daphné lui répondait de mème.(...) Tout ce temps, la fleur changeait comme si elle devait à elle seule résumer un jardin (pp. 189-190).

Ailleurs, Ana prétend que la fleur est l'âme ou le coeur de Daphné et que la fleur "se sait cueillie et à la veille de mourir" (p. 307). Le rapprochement métaphorique de la femme qui a perdu sa virginité et de la fleur cueillie est, bien évidemment, très répandu dans la littérature, mais dans Noëlle à Cuba les métamorphoses merveilleuses du laurier-rose rejoignent également l'onomastique et son intertexte mythologique. ${ }^{2 \mathrm{i}}$

Pour Pierre Léon, Icare est le personnage central de Noëlle à Cuba, "un Don Juan beau garçon mais qui fait peu la cour" (Léon, p. 93). Pourtant, le nom d'Icare le rattache non pas à la tradition littéraire du séducteur espagnol, mais à celle de la mythologie grecque. ${ }^{22}$ Ainsi, le dialogue d'Icare et de Judith dans l'avion en route pour Cuba fait allusion à l'intertexte mythologique: 
$\checkmark$ Je m’appelle Icare.

-Avec un nom pareil, vous n'avez pas peur de prendre l'avion?

-Icare, c'est un type qui n'a peur de rien.

-Icare, c'est aussi quelqu'un qui s'est brûlé les ailes.

-Je n'ai rien à perdre.

-Qu'un peu d'altitude. ${ }^{23}$

Effectivement, Icare aura au milieu du roman un accident, tombant à l'eau après avoir loué la paravoile d'un nommé Dédalo; cet accident est dû aux machinations de Chango:

- Je suis Icare, s'écria-t-il fou de joie, et je vole! Il manoeuvrait la voile maintenant comme si elle faisait partie de lui, plongeait et remontait, cédant à la griserie du cerf-volant qui ondule spasmodiquement dans les airs comme un dragon de papier. Icare volait vers le soleil, rêvait de s'y chauffer, criait "Plus haut! Plus haut!" comme s'il s'ètait senti tout près d'y toucher, alors que dans la vedette, Chango, l'amant trompé d'Ochun, un couteau à la main, sciait patiemment le câble, en jetant de temps en temps un regard au-dessus de son épaule pour s'assurer qu'on ne le voyait pas. ${ }^{24}$

Mais aussi évident que soit l'intertexte suggéré par le nom Icare, une analyse de l'onomastique dans Noëlle à Cuba se doit d'indiquer que le personnage d'Icare présente également des attributs d'au moins deux autres personnages mythologiques, Zeus et Orphée. Ce sont les prouesses sexuelles d'Icare qui le rapprochent de Zeus; Adèle et Brigitte, qui auront toutes deux des liaisons avec lui à Cuba, admirent Icare à l'aéroport de Montréal:

Un cygne se serait glissé sur un étang à canards qu'il n'aurait pas fait plus sensation qu'Icare. Tout ce qui était gris, brun ou noir parut à 
l'instant ordinaire, inélégant, ramassé. Mais personne n'eut le temps d'en souffrir car le soleil qui s'était levé pour voir, donnait à tout ce blanc l'éclat d'une épiphanie et ranimait du même coup les couleurs les plus sombres.

... Zeus en personne ou je ne m'y connais pas.

- Mauvais cygne...-Veux-tu bien t'arrêter, oiseau de malheur! Je ne suis pas en retraite; je suis en vacances! (p. 19)

La métaphore qui compare Icare à un cygne, ainsi que le jeu de langage "mauvais cygne"/mauvais signe," et le commentaire du personnage qui voit Icare comme Zeus, font appel à l'intertexte mythologique de Zeus métamorphosé en cygne pour séduire Léda. ${ }^{25}$ (Pourtant, l'intertexte de l'aviateur Icare n'est pas oublié ici, puisque l'arrivée du jeune homme est associée au soleil qui "s'était levé pour voir".) Le narrateur soulignera de nouveau l'intertexte de Léda et le cygne lorsque cette même Adèle, dont le prénom est l'anagramme de "Léda," deviendra l'amante d'Icare:

Très tard ce soir-là, quand Adèle rentra dans leur chambre, elle vit Brigitte couchée sur le côté qui lui tournait le dos et comprit qu'elle ferait mieux de rouler la chemise blanche d'Icare et la mettre au fond de sa valise, sans lui parler du cygne qui avait conquis Léda (p. 120).

Ainsi, le prénom anagrammatique d'Adèle, la première des touristes à voir Icare avant de quitter Montréal, et la première "conquise" par lui, à qui il donne une chemise blanche qui rappelle évidemment les plumes du cygne, relève de l'intertexte mythologique. D'ailleurs, l'efficacité d'Icare en tant que séducteur - on compte parmi ses amantes à Cuba la Veuve Noire, Adèle, Ochun, Liljana, Brigitte et Suzanne - le rapproche de son avatar mythologique Zeus, qui a eu pour femme neuf déesses et quinze mortelles, dont Léda. ${ }^{26}$

Mais le personnage d'Icare comporte également des traits qui le rapprochent d'Orphée, héros de la mythologie grecque. Si le prénom d'Adèle sert d'indice partiel au parallélisme Zeus/Icare, celui de 
Eurydice Branchu révèle l'Icare de Noëlle à Cuba en tant qu'Orphée. La veille de Noël, Icare emmène cette grosse femme sympathique à La Havane pour la messe de minuit, refusant de lui dire de quoi il s'agit:

Icare frappa à une porte. On lui ouvrit. Il poussa Eurydice devant lui vers une autre porte qui laissait passer un mince filet de lumière.

"Mon doux Seigneur, miséricorde! Veux-tu bien me dire où c'est qu'on se trouve, pour l'amour? Pour l'amour? Est-ce qu'il était sérieux? Je ne sais plus! Les hommes de nos jours sont bien assez fous pour faire n'importe quoil Dire que je me suis laissée emmener ici sans même offrir de résistance ... On ne peut plus se fier à personne." Elle commençait à se sentir mal à l'aise.

$\neg$ Je sais votre inquiétude, lui souffla-t-il à l'oreille.

"Ce doit être le diable en personne. Pas moyen de lui cacher quoi que ce soit, même dans le noir."

-Mais ne vous retournez pas pour me parler. Vous seriez perdue et peut-être me perdrais-je moi-même avec vous pour vous avoir conduite ici (pp. 355-356).

Cette dernière injonction, ainsi que l'atmosphère de tension et le couloir sombre qu'Icare et Eurydice doivent passer, rappellent évidemment le récit d'Orphée descendu aux enfers chercher son épouse défunte, Eurydice. ${ }^{27}$ Mais cet épisode n'est pas le seul à évoquer l'intertexte orphique. Icare présente d'autres caractéristiques de l'Orphée grec, notamment un talent musical dont il est question à plusieurs reprises.

Icare chante une "chanson de cowboy" pour ses compagnons de voyage, dans le bus qui les conduit de l'aéroport de La Havane jusqu'à leur hôtel, peu après leur arrivée (p. 45). L'incident semble banal, mais "La Ballade du cowboy de Calgary," qui sert à égayer des 
touristes fatigués et irrités après être passés à la douane, suggère encore Orphée: en écoutant ce héros grec chanter, les Argonautes oublient leur colère et leur dispute. ${ }^{28} \mathrm{Et}$ comme Orphée qui sauve les Argonautes des Sirènes, par son chant (Guthrie, p. 28), Icare trouvera sa propre Sirène, la Veuve Noire:

Tout à coup il prit à Icare une folle envie de sortir des coulisses et d'entrer sur scène, convaincu qu'il y avait dans ce drame en suspens un rôle pour lui. Le chauffeur et avec lui, Denyse, la représentante locale de Pluritour, visiblement tourmentés, voulurent lui faire comprendre les dangers de se mêler d'histoires de police. Mais plus on tentait de le dissuader, plus il lui devenait urgent d'agir. Le diable en personne n'exerça jamais autant d'attrait sur un coeur d'homme que la silhouette qu'on devinait à peine derrière les glaces teintées. Qui, mais qui, l'appelait de sa voix de sirène comme une princesse des contes, prisonnière dans une île enchantée?

- La Viuda Negra (pp. 50-51).

On constate de nouveau ici l'intertexte mythologique associé à la mise en relief du caractère artificiel, voire théâtral du rôle joué par le personnage.

En plus des multiples avatars mythologiques d'Icare, ce personnage assume lui-même à plusieurs reprises une identité ludique, héros de contes de fées ou de récit d'aventures. En plus de se présenter à Daphné comme le Chevalier au laurier-rose (p. 71), il insiste pour que Suzanne l'appelle Robinson, tandis qu'il la baptise Mercredi (pp. 298-302). A cette multiplicité d'intertextes il faut ajouter celui de la légende du joueur de flûte d'Hameln, qui aurait enlevé tous les enfants du village en jouant de sa flûte magique: Icare est un "enchanteur" aux yeux des enfants d'un village cubain, qui le suivent hors du village quand il joue de la guitare (p. 275-277). Et, aux multiples identités d'Icare, ajoutons celle de "Santiago," James Wilson Morrice, peintre qui ressemble à Icare "tellement qu'il aurait 
pu être son père" (p. 363) et aussi celle de "Bela Lugosi, dans le rôle de Dracula," dont Icare imite laccent, incarnant ainsi un acteur célèbre. ${ }^{29}$ Étant donnè les nombreux rôles intertextuels joués par Icare, rôles que le narrateur lui attribue ou que ce personnage adopte de façon ludique lui-même, il est permis de se demander pourquoi le personnage porte le nom Icare, plutôt que de s'appeler, par exemple, Orphée ou Zeus. Et c'est ici que nous identifions un dernier jeu de l'onomastique, basé cette fois-ci sur l'homonymie partielle et sur une caractéristique fondamentale partagée: il se peut bien que derrière ce personnage, le seul touriste canadien de l'Hôtel Marazul qui n'est pas québécois, mais franco-ontarien habitant Toronto, se profile discrètement l'auteur réel, non pas Icare, mais Karch. ${ }^{30}$ Ainsi Icare constitue dans Noëlle à Cuba le cas par excellence du vacancier à l'identité mouvante et problématique.

Le plaisir ludique des personnages de Noëlle à Cuba devant les multiples rôles qu'ils jouent en vacances, rôles suggèrés le plus souvent par l'onomastique, ne fait pas de doute: la nuit où Icare la prénomme Mercredi, Suzanne s'amuse "comme une enfant qui s'invente un rôle en se costumant" (p. 293). Même Daphné, qui se fait presque violer par son mari leur nuit de noces et qui a honte du "rôle" que Paul lui fait jouer le lendemain, lorsqu'il la photographie nue, se choisit alors un nouveau rôle mythologique, celui d'une vestale d'Aphrodite dansée par Isadora Duncan:

Elle était maintenant Isadora Duncan qui dansait, sur une musique douce, une danse ancienne qui avait été celle des vestales de Chypre (p. 113).

Tous ces noms ludiques et mythologiques qui mettent sans cesse l'accent sur le parallélisme de la récréation et de la re-création, proposent aussi un jeu au lecteur: si Paul est aux yeux de Daphné "un spectateur au premier rang du théâtre" (p. 114), le lecteur, qui assiste lui aussi aux multiples avatars du nom et de l'identité dans Noëlle à Cuba, se laisse séduire à son tour par le jeu de l'onomastique, le jeu des vacances et, en fin de compte, par le jeu de la lecture. 


\section{Notes}

${ }^{1}$ Agnes Whitfield, "Le Matou ontarien: les griffes d'un Pierre Karch enjôleur," Liaison, no 51 (mars-avril 1989), p. 17.

${ }^{2}$ Pierre Hébert, "L'Invitation au voyage," Lettres québécoises, no 53 (printemps 1989), p. 21.

${ }^{3}$ Pierre Karch, Noëlle à Cuba, (Sudbury: Prise de Parole, 1988), p. 161 .

${ }^{4}$ Janet Paterson identifie l'intertextualité et les jeux du signifiant comme des techniques de l'autoreprésentation au niveau du code de l'énoncé/narration ("L'Autoreprésentation: formes et discours," Texte, no 1 (1982), pp. 184-185).

${ }^{5}$ L'intérêt que porte Karch à l'onomastique et à la relation du nom et de l'identité se manifeste déjà dans son premier roman Baptême: toute l'intrigue tourne autour du conflit entre la mère qui veut baptiser sa fille "Albanie" et le curé, qui, secondé par la grand-mère de la petite, la baptise plutôt "Emma." La mère devra abandonner en fin de compte la fille mal-nommée, tout comme si l'être de l'enfant était inextricablement lié au nom.

${ }^{6}$ Eurydice Branchu est le seul personnage dont le nom figure dans deux catégories. Il faut ajouter à cette liste de personnages celui, anonyme, de l'écrivain, ainsi que dix touristes dont le nom ne relève pas d'un intertexte mythologique, ni ne présente un jeu de langage (Ana, Brigitte, Céline, David, Hubert, Jean-Marc, Judith, Liljana, Suzanne, Sylvio).

${ }^{7}$ On trouve, en plus de ces personnages cubains, la figure importante de la "petite vieille" qui prend Icare pour James Wilson Morrice, et son fils, également anonyme, ainsi que plusieurs personnages à prénom non-parlant (Carlos, Manuel, Marco, Ricardo, Rosa, Teresa, Wifrido). Il y a d'autres personnages cubains anonymes et épisodiques, tels qu'un chauffeur de bus et des groupes d'enfants au marché et au village d'Ernest Hemingway. 
${ }^{8}$ Philippe Hamon, «Pour un statut sémiologique du personnage," Gérard Genette, Tzvetan Todorov, éd., Poétique du récit (Paris: Seuil, 1977), p. 145.

${ }^{9}$ Pierre Léon, "Noëlle à Cuba: un roman de la modernité," LittéRéalité, vol. I, no 1 (printemps 1989), p. 92.

${ }^{10}$ Noëlle à Cuba, p. 385. D'autres noms de famille jouent de façon plus discrète sur le langage. François Lannes est un personnage impuissant qui aime les animaux mais travaille au laboratoire de neuropathologie de l'Université McGill, où les animaux dont il s'occupe sont des sujets d'expériences scientifiques. Le nom de ce personnage "trop délicat pour être palefrenier et trop sensible pour être vétérinairen (p. 14), suggère-t-il peut-être l'âne et aussi l'animal? De même, le nom du bon vivant Antonio Vervier évoque la verve caractéristique de cet animateur du groupe. Les seuls noms de famille qui restent, ceux de Roland Rolland et d'Ian McDonald, ne relèvent pas d'un jeu de langage. Le fait que Roland porte un prénom et un nom de famille identiques ne fait que rendre ce personnage un peu ridicule, tandis que le nom d'Ian est typiquement écossais, ce qui le distingue des autres personnages sans lui attribuer d'autres caractéristiques individuelles.

${ }^{11}$ Raymond W. Thorp et Weldon D. Woodson, The Black Widow Spider (New York: Dover Publications, 1976), p. 100.

${ }^{12}$ Tzvetan Todorov note qu'il y a souvent dans le récit fantastique une équivalence entre l'amour et la mise à mort, où le corps désirable se trouve rapproché du cadavre IIntroduction à la littérature fantastique (Paris: Seuil, 1970), pp. 141-143]. Karch est également l'auteur d'un recueil de contes fantastiques: Nuits blanches (Sudbury: Prise de Parole, 1981).

${ }^{13}$ Ruth Amossy et Elisheva Rosen notent que le but du texte qui effectue de façon systematique une pratique ludique du cliché est de distraire et aussi «à travers ce 'plaisir du texte' très particulier, de promouvoir une réflexion sur le langage en général et sur le discours romanesque en particulier" [Le Discours du cliché (Paris: Sedes, 
1982), p. 111]. Ces commentaires s'appliquent fort bien à Noëlle à Cuba, non seulement en ce qui concerne le cliché transformé de façon ludique par Mariposa, mais aussi pour ce qui est de l'onomastique ludique, signe de l'écriture.

${ }^{14}$ Noëlle à Cuba, p. 168. Nous verrons en analysant les noms mythologiques de Noëlle $\grave{a}$ Cuba que le concept d'ailes convient également au personnage Icare, qui s'inspire nettement de l'aviateur mythologique du même nom et de Zeus métamorphosé en cygne pour séduire Léda.

${ }^{15}$ Les métamorphoses possibles de Mariposa en papillon relèvent bien du fantastique tel que défini par Todorov, qui insiste sur l'hésitation du lecteur *entre une explication naturelle et une explication surnaturelle des événements évoqués" (Todorov, p. 37).

${ }^{16}$ Le phénomène du nom mythologique dans Noëlle à Cuba reflète peut-être l'intérêt que porte Karch à l'oeuvre de Maurice de Guérin, auteur dont La Bacchante et Le Centaure présentent des personnages mythologiques. Hédi Bouraoui et Jacques Flamand notent que Karch est l'auteur de plus de quarante articles sur les Guérin [Écriture franco-ontarienne d'aujourd'hui (Ottawa: Vermillon, 1989), pp. 161-162].

${ }^{17}$ Louis Maximilien note que le dieu Schango, ou Ogoun Schango, est l'une des plus puissantes divinités de la mythologie vaudoue de Haït, dieu qui a fait place au Christ en Haïti. Schango avait pour épouses "Oya, Oschoun et Oba, les trois déesses de l'eau," tandis que l'Ochun de Noëlle à Cuba est l'amante de Chango. Maximilien précise que la déesse Oschoun se retrouve à Cuba [Le Vaudou haïtien (Port-au-Prince: Imprimerie de l'Etat, 1944), p. 12-13]. Joseph J. Williams indique lui aussi les nombreux liens entre la mythologie afro-cubaine et celle de Haïti [Voodoos and Obeahs: Phases of West Indian Witchcraft (New York: Dial Press, 1933), pp. 82-83].

${ }^{18} \mathrm{~A}$ un autre niveau d'analyse, l'on peut se demander si les deux affrontements de Chango et d'Icare, divinité afro-cubaine et héros de l'Antiquité grecque, d'où Icare sort vainqueur (pp. 197; p. 240-241), 
reflète en microcosme la rencontre des touristes venus du pays riche et "civilisé" et des Cubains, citoyens d'un pays en voie de développement.

${ }^{19}$ Marianne Clouzot, èd., Les Métamorphoses d'Ovide (Paris: Sodac, 1947), pp. 85-91.

${ }^{20}$ Le procédé employé ici rappelle ceux identifiès par Gérard Genette et Jean Ricardou. Genette décrit l'importance des relations de contiguïté dans l'exercice du rapport métonymique chez Proust, où des objets contigus subissent un rapprochement analogique, tandis que Ricardou identifie un "fantastique de l'écriture" qui a lieu grâce à la métaphore structurelle [Genette, Figures III (Paris: Seuil, 1972), pp. 41-63; Ricardou, Problèmes du nouveau roman (Paris: Seuil, 1967), p. 136].

${ }^{21}$ A un moment donné, le laurier-rose semble se venger du mari ègoïste de Daphné: après avoir gêné sa femme en la photographiant nue, Paul trébuche sur un tronc de laurier-rose et tombe à la renverse dans la mer (p. 114).

${ }^{22}$ Pour l'histoire de la fuite de Dédale et la mort d'Icare, voir Jacques Desautels, Dieux et mythes de la Grèce ancienne (Québec: Presses de l'Universitê Laval, 1988), pp. 430-431.

${ }^{23}$ Noëlle à Cuba, p. 28. De même, Suzanne dira à Icare "Tu es un mythe, Icare" (p. 302).

${ }^{24}$ Noëlle à Cuba, p. 235. La prévisibilité du programme narratif n'est pourtant pas entière ici, dans la mesure où l'Icare de Karch ne meurt pas.

${ }^{25} \mathrm{Ce}$ jeu de langage, comme celui fait par Mariposa qui voit en Icare "une autre paire d'ailes" (p. 105), sert également à surdéterminer la dimension "aérienne" de l'aviateur mythologique qu'est Icare.

${ }^{26}$ Desautels, Dieux et mythes de la Grèce ancienne, p. 156. 
${ }^{27}$ Pour le récit de la descente d'Orphée aux enfers, voir Desautels, pp. 547-548.

${ }^{28}$ W.K.C. Guthrie, Orpheus and Greek Religion: A Study of the Orphic Movement (London: Methuen, 1952), p. 28.

${ }^{29}$ Noëlle à Cuba, p. 295. Le roman peint souvent des personnages qui jouent consciemment un rôle, procédé qui seconde la dimension autoreprésentative de l'onomastique. Parmi les meilleurs exemples de ce phénomène signalons le passage où Icare en compagnie de la Veuve Noire se demande "quel rôle on lui réservait dans cette histoire" (p. 93), celui où Icare, "qui aimait au théâtre les scènes où l'on se parle à genoux," se jette aux pieds d'un autre personnage (p. 156), et celui qui précise que Mariposa "s'inventait un personnage" chaque fois qu'elle ètait en présence d'Icare, "comme si elle se cherchait un rôle dans le rêve qu'il se fabriquait" (p. 267). En fait, c'est Icare, le personnage le plus complexe du roman, qui réfléchit constamment au théâtre et à son propre rôle: on trouvera d'autres exemples de ce phénomène chez Icare aux pages 169 et 296.

${ }^{30}$ Paterson note que la création "d'un personnage écrivain ou d'une figure auctoriale relève d'une si longue tradition littéraire qu'il est aisé d'y voir un reflet de la création artistique" (Paterson, p. 179). L'autre contrepartie romanesque de Karch serait sans doute l'ècrivain anonyme qui visite Cuba, surtout étant donné le fait, noté par Léon et Hébert, que la technique de Karch dans Noëlle à Cuba ressemble à celle décrite par ce personnage (Léon, p. 92; Hébert, p. 21). 